\title{
Sacral insufficiency fracture following rectal cancer treatment - case report
}

\author{
Agnieszka Pikuła ${ }^{1, D \oplus}$, Tomasz Stanisław Pikuła ${ }^{2, D \oplus}$, Mateusz Pastuszak ${ }^{3, D \odot}$ \\ ${ }^{1}$ Department of Surgical Oncology, Medical University, Lublin, Poland \\ ${ }^{2}$ Interventional Radiology and Diagnostic Imaging Department, Public Teaching Hospital No. 1, Lublin, Poland \\ ${ }^{3}$ Medical University, Warsaw, Poland \\ A - Research concept and design, B - Collection and/or assembly of data, C - Data analysis and interpretation, \\ $D$ - Writing the article, E - Critical revision of the article, F- Final approval of article
}

Pikuła A, Pikuła TS, Pastuszak M. Sacral insufficiency fracture following rectal cancer treatment - Case study. J Pre-Clin Clin Res. 2020; 14(4): 120-122. doi: $10.26444 / j p c c r / 128136$

\begin{abstract}
Introduction and Objective. Locally advanced rectal cancers witha high risk of recurrence need multimodal treatment Rusing neoadiuvant preoperative chemoradiotherapy or preoperative radiotherapy. Both rectal cancer surgery and the additional chemoradiotherapy or radiotherapy may cause late sequelae. The aim of the study is to present a clinical situation in which a pelvic recurrence of rectal cancer has to be distinguished from therapy side effects. Insufficiency fracture (IF) is one of the therapy side-effects. It can cause pain and decrease mobility and it is a well-known late complication to pelvic radiotherapy, but can be misinterpreted as a local recurrence.

Conclusions. It is extremely important to distinguish IF from metastases, which may require biopsies and initiation of potentially toxic treatments such as chemotherapy. MRI and CT scans are complementary modalities to make an accurate diagnosis of IF.
\end{abstract}

\section{Key words}

rectal cancer, sacral fracture, Insufficiency fracture

\section{INTRODUCTION}

The incidence of rectal cancer in the European Union is estimated at 125,000 cases annually, with an increasing tendency in both males and females. Mortality varies from 4-10 cases per 100,000 per year and the median age at diagnosis is $\sim 70$ [1]. Rectal cancer treatment depends on the disease severity and is risk-adapted. Very early tumours with clinical stage T1N0, with low grade (G1/G2), can be treated by local excisional procedures such as transanal endoscopic microsurgery $[2,3]$. Higher stages or early rectal cancer not suitable for local excision should be treated by radical total mesorectal excision (TME) surgery [2]. Locally advanced rectal cancers with high risk of recurrence need multimodal treatment with the use of neoadjuvant preoperative chemoradiotherapy or preoperative radiotherapy $[2,3]$. Both rectal cancer surgery and the additional radiotherapy or chemoradiotherapy may cause late sequelae, which impact on daily activity. Long-term side-effects of treatment should be controlled. Radiotherapy for rectal cancer may cause significant long-term lower genitourinary toxicities and fecal incontinence[4].

Patients suffering from faecal incontinence have a poorer quality of life. Occurring pain (as a symptom decreasing life quality) should be analyzed as a side-effect as well as a possible sign of disease recurrence.

Address for correspondence: Agnieszka Pikuła, Department of Surgical Oncology, Medical University, Radziwiłłowska 13, 20-080, Lublin, Poland

E-mail: pastuszek@interia.pl

Received: 28.06.2020; accepted: 05.10.2020; first published: 22.10 .2020

\section{OBJECTIVE}

The aim of the study is to present the clinical situation in which a pelvic recurrence of rectal cancer has to be distinguished from therapy side-effects.

\section{CASE REPORT}

A 66-year-old female patient was admitted to hospital due to rectal bleeding. She was in good performance status, with no comorbidities and no weight loss. Colonoscopy, thoracic $\mathrm{x}$-ray, abdomen ultrasound and computed tomography (CT) of abdomen and pelvis were performed. Full blood count, liver and renal function tests, serum carcinoembryonic antigen (CEA) were also assessed. There were no clinically significant abnormalities beside irregular circular thickening of the rectal wall in computed tomography. Colonoscopy revealed circular infiltration localized about $10 \mathrm{~cm}$ from the anal sphincter. Histopathological assessment of tumour specimens confirmed the diagnosis of rectal adenocarcinoma G2. The patient was referred to the oncologic surgery unit. Magnetic resonance imaging (MRI) of the pelvis was performed revealing a pathological infiltration about $8.5 \mathrm{~cm}$ from the anorectal junction. The tumour measured $4 \mathrm{~cm}$ in length and occupied $2 / 3$ of the perimeter of the rectal wall and infiltrated the mesorectum up to $5 \mathrm{~mm}$. The circumferential resection margin (CRM) was not involved. Clinical staging was T3b CRM (-) N0. The patient was referred to preoperative radiotherapy with a 25 Gy total dose at 5 Gy/fraction during 5 days. Eight weeks after radiotherapy rectal cancer surgery - total mesorectal excision - was performed. Result of the histopathological examination confirmed diagnosis of rectal adenocarcinoma and assessed the quality of surgery 
as good, with no nodal involvement and moderate response to treatment, with tumour down-staging to yT2N0. There were no early postsurgical complications; however, mild chronic diarrhea occurred after radiotherapy.

Eight months after surgery, a follow-up computed tomography was performed revealing no metastases; CEA was also normal. Two months later, the patient complained of pain localized in the sacral area; she denied mobility impairment. There was no medical history of injury in this area. CEA was still within normal range. MRI of the pelvis was recommended and revealed a fracture line, oedema and extensive enhancement in the sacrum on the right (Figs. 1,2) without restricted diffusion (Figs. 3, 4). There was a similar milder lesion on the left. It was diagnosed as sacral insufficiency fracture. Conservative treatment was performed (analgesic, calcium and vitamin D supplementation). The pain was resolved after 5 months. At that time, thoracic, abdomen and pelvic CT, as well as colonoscopy, revealed no disease dissemination / recurrence. After a 2-year follow-up, the patient is in good performance status with no symptoms of cancer.

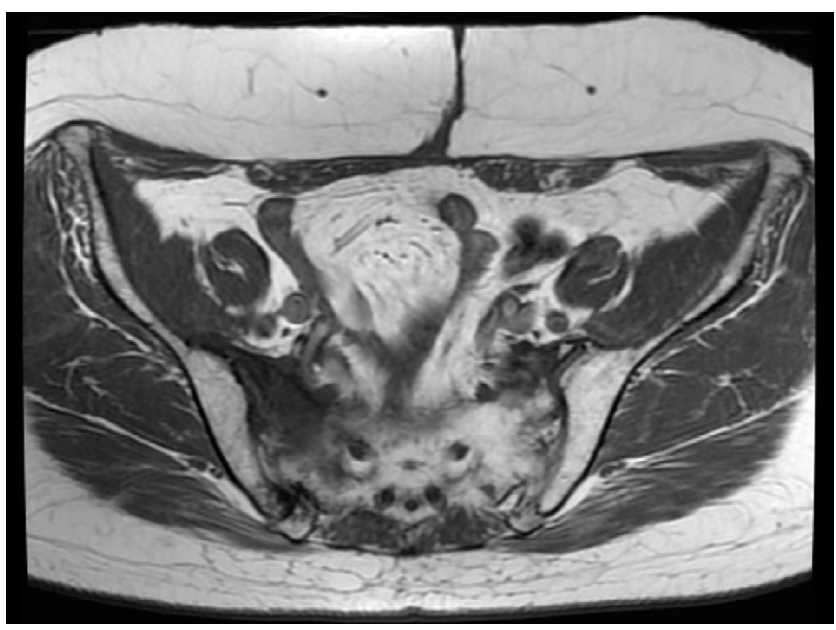

Figure 1. T1-weighted image showing hipointensity in the sacrum on the righ corresponding to fracture line and edema

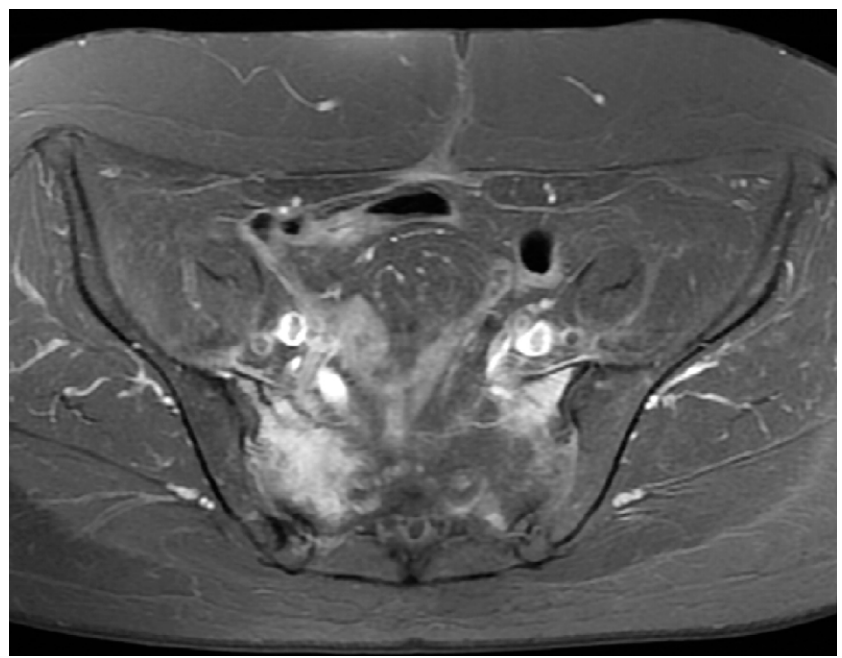

Figure 2. Post-contrast T1-weighted image with fat saturation showing hipointens fracture line and extensive enhancement in the sacrum on the right; similar milder findings are seen on the left

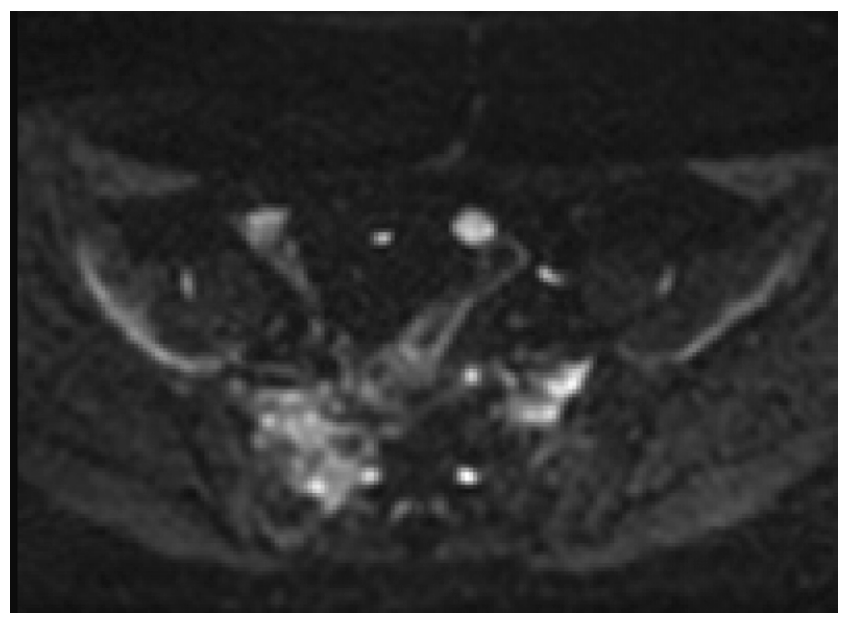

Figure 3. $\mathrm{DWI}, \mathrm{b}=800$, image showing hiperintensity in the sacrum on both sides (right > left) corresponding to edema

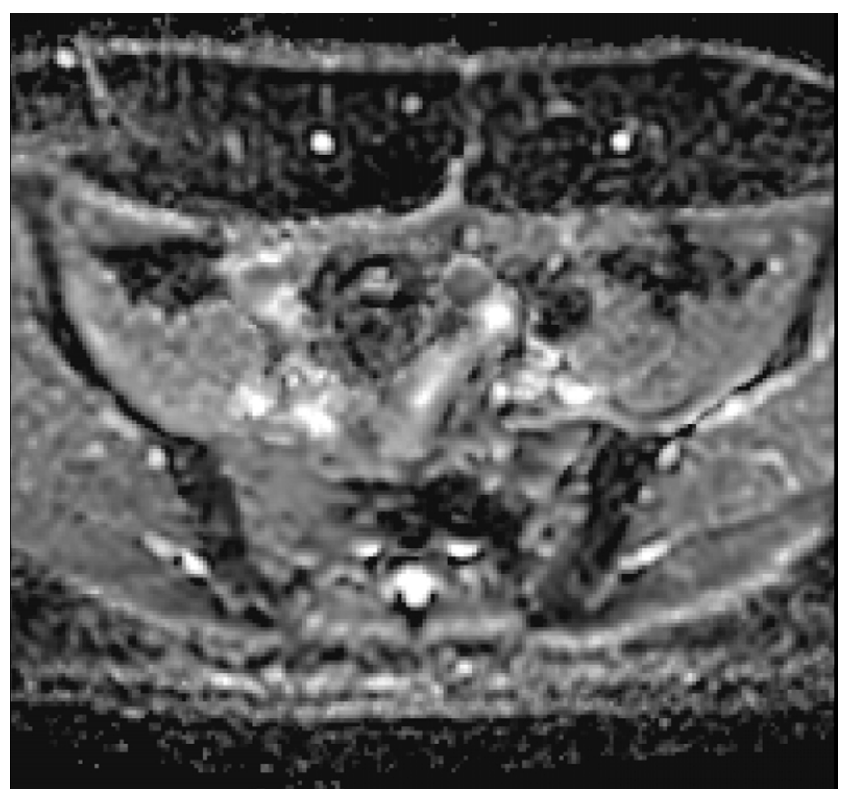

Figure 4. ADC map showing hiperintensity in the sacrum on both sides (right > left), which means that there is no diffusion restriction typical of metastatic process

\section{DISCUSSION}

Insufficiency fracture (IF) is a type of stress fracture, when normal or physiological stress is applied to weakened bones with decreased elastic resistance. It is sometimes confused with fatigue fracture, another type of stress fracture, which occurs when extreme pressure is placed on normal bone. Various circumstances can impair bone strength. Risk factors most frequently associated with IF include age, gender (female), osteoporosis, menopause, radiation dose, chemotherapy and body weight. Many studie have reported the development of IF after radiation therapy (RT) in gynaecological, prostate, anal and rectal cancer patients [4]. To make a correct diagnosis it is necessary to perform a clinical assessment accompanied by diagnostic imaging. After rectal cancer treatment patients should remain in follow-up to control long-term implications, and to detect disease recurrence. There are recommendations for regular clinical examination: colonoscopy with resection of colonic 
polyps, pelvic imaging using MRI, for distant metastases CT of the chest, abdomen and pelvis, and regular serum CEA tests. Especially high-risk patients with the involvement of circumferential resection margin can benefit from this more proactive surveillance for local recurrence [1]. The standardization of surgery and the neoadjuvant treatment allowed for the decrease of local recurrence of the disease. The innovative approach for treating rectal cancer, i.e. neoadjuvant treatment modalities and total mesorectal excision (TME), have further improved local control [5].

Pelvic insufficiency fracture has similar prevalence as local recurrence. It is challenging to distinguish between these cases. Pelvic insufficiency fracture is detected in $3.3-7.1 \%$ of cases after radiotherapy, and can be noted between 2 months to 8 years after treatment [4]. A study performed by Yu-Mei Kang et al. [6], confirmed that pelvic fracture risk is higher after 2-4 years of follow-up after RT. On this case, it is advised that physicians pay more attention to pelvic fractures during the first four years of follow-up among rectal cancer patients who receive pelvic RT. Pain is the most common symptom of post-RT pelvic fracture whilst around $20 \%-50 \%$ of people are asymptomatic [6].

Diagnostic imaging is necessary to establish the correct diagnosis. Plain radiographs are not recommended as they can delay diagnosis due to difficulties caused by image interpretation [7]. Radiotherapy-induced IF and bone metastasis show an increased uptake on a bone scintigraphy after radiotherapy' but the typical ' $H$ ' sign of IF does not always appear, which can be misinterpreted as bone metastasis [8]; therefore, bone scintigraphy should not be the first choice.

The single institution observational study showed that the most commonly observed fracture site was the sacrum, corresponding to the site of force transfer from the vertebral column to the pelvis. Morphology of insufficiency fractures is characteristic, with a generally symmetrical (H-shaped) appearance, with no mass effect. On MRI, IF presents an easily recognizable oedema signal in contrast to metastases that disorganizes the bone and forms a real replacement tissue. Moreover, radiation-induced fractures are not associated with any invasion of adjacent soft tissues, which is frequently observed in case of metastases. It is therefore essential to distinguish radiation-induced fractures from metastases, which may require biopsies and initiation of treatments such as chemotherapy. These two lesions can generally be distinguished by alternately performing pelvic CT and MRI follow-ups [9]. MRIs can detect early changes of sacral insufficiency with a proved sensitivity at or near $100 \%$. It is now established that MRI scans are most useful in identifying insufficiency fractures of the sacrum, and that their use is a gold standard [8].

\section{CONCLUSION}

Neo-adjuvant chemoradiotherapy (CRT) is an integral part of the standard treatment for advanced primary rectal cancers as it decreases the local recurrence rate. Pelvic radiotherapy may be the cause of both acute and late toxicities, including intestinal, urogenital and bone marrow affection, and weakened bone structure. IF is a type of stress fracture that occurs when physiological stress is applied to weakened bone. It can cause pain and decrease mobility and is a wellknown late complication to pelvic radiotherapy. However, it can be misinterpreted as local recurrence. Improvement in radiotherapy technique reduces the risk of IF in rectal cancer survivors [6]. MRI scans are recommended to make accurate diagnosis of IF.

\section{REFERENCES}

1. Glynne-Jones R, Wyrwicz L, Tiret E, et al. Rectal cancer: ESMO Clinical Practice Guidelines for diagnosis, treatment and follow-up Ann Oncol. 2017; 28: iv22-iv40. https://doi.org/10.1093/annonc/mdx224

2. Junginger T, Goenner U, Hitzler M, et al. Long-term oncologic outcome after transanal endoscopic microsurgery for rectal carcinoma. Dis Colon Rectum. 2016; 59: 8-15.https://doi.org/10.1097/DCR.0000000000000509

3. Stornes T, Wibe A, Nesbakken A, et al. National early rectal cancer treatment revisited. Dis Colon Rectum. 2016; 59: 623-629. https://doi. org/10.1097/DCR.0000000000000591

4. Kronborg C,Jørgensen Petersen J, Jensen L, et al. Pelvic insufficiency fractures, dose volume parameters and plan optimization after radiotherapy for rectal cancer. Clin Transl Radiat Oncol. 2019 Sep 10; 19: 72-76. https://doi.org/10.1016/j.ctro.2019.09.001

5. Ikoma N, You YN, Bednarski BK, et al. Impact of Recurrence and Salvage Surgery on Survival After Multidisciplinary Treatment of Rectal Cancer. J Clin Oncol. 2017; 35: 2631. https://doi.org/10.1200/jco.2016.72.1464

6. Kang Y, Chao T, Wang T, et al. Increased risk of pelvic fracture after radiotherapy in rectal cancer survivors: A propensity matched study. Cancer Med. 2019 Jul; 8(8): 3639-3647. https://doi.org/10.1002/ cam4.2030

7. O'Connor T, Cole P. Pelvic Insufficiency Fractures Geriatr Orthop Surg Rehabil.2014Dec;5(4): 178-90.https://doi.org/10.1177/2151458514548895 8. Zhong X, Li J, Zhang L, et al. Characterization of Insufficiency Fracture and Bone Metastasis After Radiotherapy in Patients With Cervical Cancer Detected by Bone Scan: Role of Magnetic Resonance Imaging. Front Oncol. 9: 183. https://doi.org/10.3389/fonc.2019.00183. https:// www.frontiersin.org/article/10.3389/fonc.2019.00183

9. Bazire L, Xu H, MD, Foy JH, Amessis M, Malhair C, et al. Pelvic insufficiency fracture (PIF) incidence in patients treated with intensitymodulated radiation therapy (IMRT) for gynaecological or anal cancer: single-institution experience and review of the literature Br J Radiol. May 2017; 90(1073): 20160885. https://doi.org/10.1259/bjr.20160885 